Hev. Chil. Pedietr. 68 (2); 78-82, 1997

\title{
Diálisis peritoneal crónica infantil: estado actual en Chile
}

\author{
Felipe Cavagnaro S.M. ${ }^{1}$; Edda Lagomarsino F. ${ }^{1}$; \\ Rama de Nefrología ${ }^{2}$
}

\begin{abstract}
Resumen
La diálisis pertoneol crónica en pediatria ha tenido un rópido desarrollo en los ùltimos 18 meses en Chile, en gran parte debido a políticas gubernamenlales de apoyo a niños partadcres de problemas renales crónicos lerminales. Fáa conocer la situcción actual de los pregramas que realizan este pocedimiento y los pacientes incluidos en ellos. se analizó el resultado de una enclesta enviada a tocias las inslituciones de salud en que se realizaba este lipo de diálisis en niños, entre enero de 1995 y abril de 1996. Se reunió ura población de 34 niños en diálisis periloneal crónica, prcver enles de 8 instituciones de solud. Estos pacienles presentaron lasos de complicaciones comparables a las descritas en subicaciones internacionales, a excepción de peritonitis, en la que, por el momento, se registron peoses resultados que en experiencias de pa'ses desarrollados. El procedimiento no sido bien aceplado en nuestro pais. constituyendo un claro aponte en el tratamiento de niños con uremia crónica lerminal.
\end{abstract}

(Palabras clave: diá isis peritoneal crónica, insuficiercia renal crónica, niños.)

\section{Pediatric chronic peritoneal dialysis in Chile}

Chronic perikoneal diclysis in pediatric patients has beer increasingly applied in the lasl 18 months in Chile, since fincncial support wos provided by governmental previssional lows in favor of children wilh erd stage renal disease. The curent state of chro-ic peritoned dialysis programs in the country was surveved through a questionary sent lo all puslic in = 5] and privale inslitu'ions $\left(r_{i}=3\right.$ ) which to date had active chronic periloneal dialysis programs for children between january 1995 and ap it 1990 . A tolal of 34 children were under the procedure by that lime. The causes of chrenic renal cailure were renal dysplasia (n: 9; $26 \%$, hemoytic uremic syndrcme in: $8 ; 24 \%$ ), primary glamerular disease In: 5 ; $15 \%$, reflux nephropothy in. $4 ; \mid 2 \%$, obslructive urcpothy $\{n ; 2 ; 6 \%$, one case each of Fanconi's, Alport's and prune befly syndromes and of contical nectrosis. in the last iws patients the cause was undetermined as o consecuence of severe renal atroghy at initial consultation. Complicosion rates were comparable to those reporled by 'nternaciona! publications except lo: peritonitis, whose incidence was clecrly higher icne episode per eight potients per month| than that repcrled in USA and Europe.

(Key words: chronic peritoneal diaysis, chronic renal failure, children.l

Con la incorporación de la diálisis peritoneal crónica (DPC) en niños menores de 12 años entre las prestaciones del Fondo Nacional de Salud (FONASA) de Chile', se inició en el país un rápido auge en la aplicación del procedimiento a un segmento de la población pediátrica portado-

1. Departamento de Pediatria, Pontificia Universidad Católica de Chile.

2. Sociedad Chilena de Pediatría. ra de insuficiencia renal crónica (IRC) terminal que, en la mayoría de los casos no estaba recibiendo otro tipo de terapia de sustitución renal. Con posterioridad, apoyándose en el buen resultado de lo anterior, se extendió la cobertura para DPC en el resto de la población ${ }^{2}$, incorporando así a niños mayores de 12 años y asegurando su permanencia en programas de díalisis a largo plazo.

Con el propósito de describir el estado actual de los programas de DPC en niños, las caracte- 
rísticas de los pacientes, y eventualmente sugerir estándares de manejo para que mejoren su eficiencia y eficacia, se solicitó y analizó información de los casos registrados y controlados en diferentes centros del país.

\section{Método}

Con el auspicio de la Rama de Nefrología de la Sociedad Chilena de Pediatria, se confecciono un cuestionario que fue enviado a lus encargados de los programes de DPC en niños en cada una de las instituciones públicas (hospitales Luis Calvo M., Roberto del Río, Exequiel González C., San Juan de Dios, todos de Santiago. y Regional de Antofagasta) o privadas (Hospital Clínico de la Universidad Católica, clínicas Las Condes e Indisa, todos de Santiogol en que el procedimiento se realizaba, solicitando información sobre los pacientes que se estuviesen beneficiando de él entre el 1 de enero de 1995 y e] 31 de abril de 1996. incluyendo sus características demogróficas, antropométricas y previsionales: cousa y tratamiento recibido por la insuficiencia renal crónica; características de la DPC y sus complicaciones: evolución en el mediano plazo y eslado de salud actual de los afeclados. En el análisis de las medidas corporales se emplezron los estándares de las tablas del Cenıro Estadounidenşe de Estadísticas de Salud (NCHS) para edad y sexo.

\section{Resultados}

Los ocho centros encuestados enviaron sus respuesta, que incluian un total de 34 nir̃os ( 20 niñas), la mediana de cuyas edades en el momento del estudio era 8,9 años (márgenes 1,2 y 21 años), con la distribución que muestra la figura 1. La causa de la IRC fue: displasia renal en $9(26 \%)$. síndrome hemolítico urémico en 8

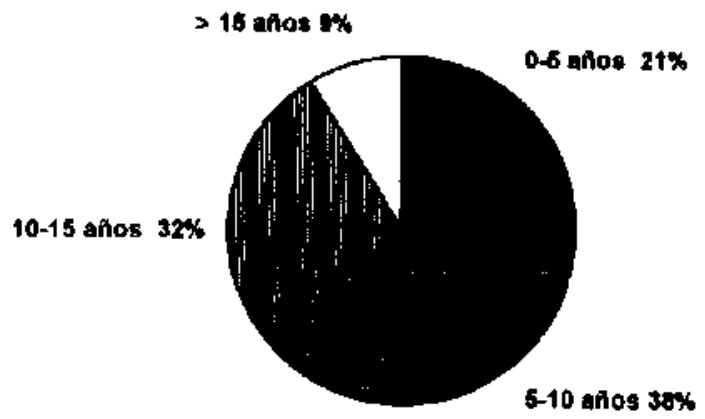

Figura 1: Distribución por edad de 34 pacientes pediaritos en programas de dílisis peritoneal crónica en ocho centros chilenos.

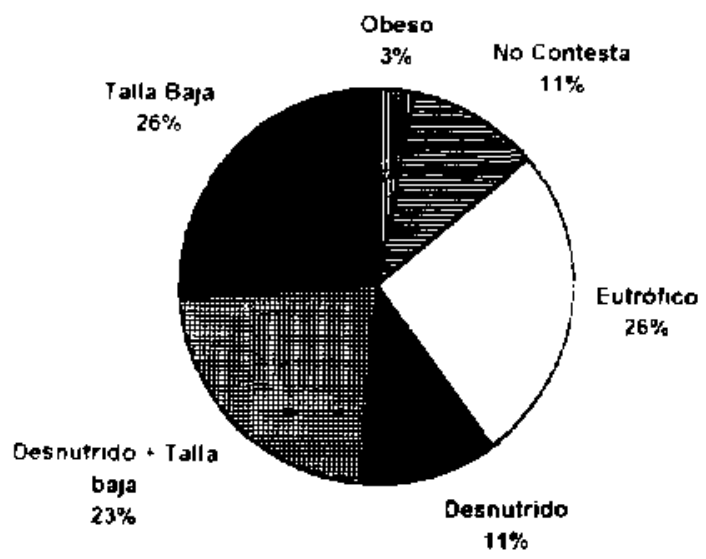

Figura 2: Situación nutricional en 34 pacientes de centros chilenos al comienzo de la diálisis peritoneal cronica.

(24\%), glomerulopatía primaria en 5 (15\%) nefropatía por reflujo en $4(12 \%)$, uropatía obstructiva en $2(6 \%)$. Se registró además un caso en cada uno de los síndromes de Fanconi, Alport y abdomen en pruna y otro de necrosis corticat. En dos pacientes no se conocía la causa de la insuficiencia renal, en ambos el síndrome urémico fue la primera manifestación de la afección, y tenían atrofia renal de tal intensidad que no permitía hacer una aproximación al diagnóstico etiológico. El diagnóstico de insuficiencia renal se hizo a la edad (promedio) de 5,5 años (márgenes 0 y 13 años), y la DPC comenź (promedio) a los 8,2 años (márgenes I a 16,I años). En siete pacientes ( $20 \%$ ) se había realizado algún tipo de procedimiento previo para reemplazar por tiempo prolongado la función renal: en cinco un trasplante renal y en dos diálisis peritoneal aguda prolongada (más de 3 semanas). Veintisiete niños (79\%) eran beneficiarios del sistema de previsión de salud estatal (FONA-SA), Ios demás de instituciones previsionales privadas (ISAPRE), 20 (59\%) vivían en la Región Metropolitana, siete en regiones del sur, seis en el norte y uno en la costa central del país.

La figura 2 muestra que más de la mitad de los pacientes tenía estatura menor al percentil 5 para edad y sexo y un subgrupo sufría, además, relación peso/talla menor al percentil 5.

La inserción de catéter peritoneal habfa sido, en todos los casos, realizada en pabellón por un cirujano o urólogo pediátrico, empleando en su totalidad catéteres tipo Tenckhoff con extremo 
distal en espital en $59 \%$ de los casos y doble manguito en $63 \%$. Si bien en todas las instala. ciones se dejó un trayecto en túnel subcutáneo, cl sitio de salida escogido fue variable (figura 3). El tiempo transcurrido entre la colocación del catéter y el inicio de la DPC varió entre 0 y 21 días, promedio 6.7 días. Es importante mencronar, para efectos de consideraciones de inciusión a la DPC, que en 17 pacientes ( $58 \%$ ) había antecedentes de cirugía abdominal previa (figura 4), incluyendo trasplante renal, lo que, salvo en un caso, no representó más problemas para la diálisis que los de aquéllos sin cirugía previa. En nueve pacientes $(26 \%)$ fue necesario cambiar el catéter peritoneal en al menos una oportunidad, principalmente debido a problemas de flujo o infección recurrente.

Sólo en tres pacientes se empleaba, además de la diálisis peritoneal crónica ambulatoria, otro tipo de terapia: dos DPC intermitente y uno DPC automatizada cíclica. La mayoría $(82 \%)$ sólo utilizaba solución "isotónica" de diálisis con glucosa al 1,5\%: sólo en seis enfermos se requerian mezclas con soluciones más concenIradas (2.5\% y $4,25 \%$ ). Veintidós pacientes, casi dos tercios de los ellos, requerian cuatro ciclos de diálisis diarios; el otro tercio se repartio entre tres ( 5 pacientes), cinco ( 5 pacientes) y seis ciclos ( 2 pacientes). En 19 casos (56\%) se había realizado un estudio de adecuación de diálisis (prueba de equilibrio peritoneal) en una etapa temprana de su incorporación a DPC. En 15 pacientes $(44 \%)$ se usaba eritropoyetina humana recombinante. La satisfacción del equipo tratante sobre los resultados del procedimiento eran superiores a $90 \%$ en lo que respecta al control de productos nitrogenados, equilibrio electrolítico y de ácidos y bases y control de la volemia.
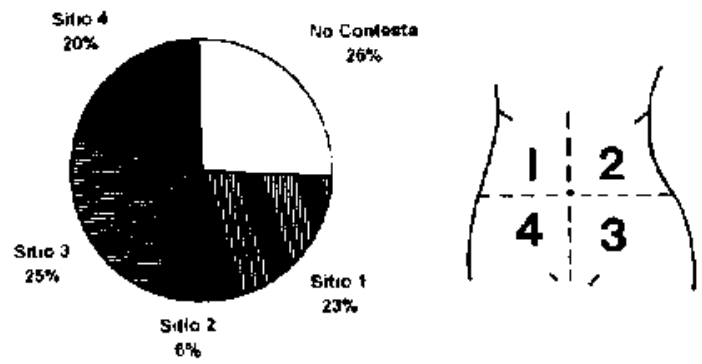

Figura 3: Ubicación de la salida del catéter periloneal en 34 pacientes en diálisis crónica peritoneal.

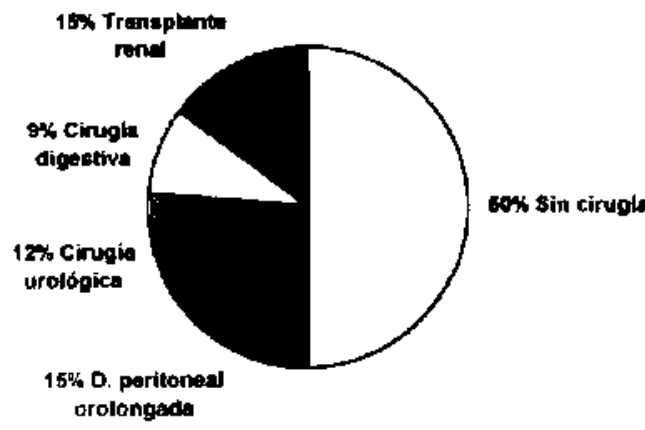

Figura 4: Antecedentes de cirugía abdominal previa a la diálisis crónica peritoneal en los 34 pacientes de la serie

Se contabilizaron 34 episodios de peritonitis agudas -2 de ellas recurrentes- que afectaron a 16 pacientes; esto dio una tasa de aproximadamente un episodio de peritonitis cada 8 pacientes por mes. Los microorganismos más frecuentemente aislados fueron Staphylococcus aureus (38\%) y Staphylococcus epidermidis (18\%); in $29 \%$ de las peritonitis no se aisló gérmenes en el cultivo de líquido peritoneal. Otras complicaciones microbianas fueron infección del sitio de salida 0 en el túnel subcutáneo en 12 y 2 pacientes respectivamente. No hubo correlación entre el sitio de salida del catéter y la incidencia de complicaciones infecciosas.

S6lo en 4 pacientes $(11,7 \%)$ se dio término al tratamiento con diálisis peritoneal crónica durante el trascurso de esta evaluación: uno por fallecimiento debido a bronconeumonía, otro por trasplante renal, uno por cambio a hemodiálisis, y otro por mejoría de su función renal.

En términos de escolaridad, 22 de $\operatorname{los} 23$ pacientes en edad escolar ( $>6$ años) asistían regularmente a un establecimiento educacional, si bien el rendimiento escolar no fue evaluado en este estudio. El único paciente que no asistía sufre de una enfermedad psiquiátrica crónica (esquizofrenia).

\section{Comentario}

El rápido desarrollo de la DPC pediátrica en nuestro país ha constituido, sin lugar a dudas, un factor trascendental en el mejor pronostico vital de un importante número de niños portado- 
res de IRC terminal. Experiencias pediátricas nacionales publicadas anteriormente ${ }^{3,4}$ ya avalaban esta técnica como una opción promisoria en niños afectados por esta patología.

-Más de $20 \%$ de los pacientes registrados en este estudio tentan menos de 5 años de edad, subgrupo que tradicionalmente sufría de alta morbilidad y mortalidad, pues el tratamiento altemativo, la hemodiális, es técnicamente muy compleja en ellos. La baja proporción de pacientes mayores de 15 años se debe, principalmente. a que en su mayoría son atendidos por programas de salud de adultos, en los que, al menos por el momento, se favorece la hemodiálisis crónica como opción.

La distribución de las causas de la IRC en los pacientes de esta serie es diferente de la descrita en otras estadísticas nacionales ${ }^{5,6}$, donde las uropatías obstructivas aparecen como la principal, seguidas de hipoplasias y displasias, glomerulopatías y nefropatía del reflujo. Es posible que para algunos pacientes con malformaciones urinarias obstructivas sometidos a muchos procedimientos quirúrgicos desobstructivos o correctores, pudiese haberse estimado que el método era poco apropiado, pues es más efectivo si la membrana peritoneal está indemne. El gran número de pacientes con IRC debida a síndrome hemolítico urémico tampoco concuerda con los estudios nacionales antes citados, y no tenemos explicación para ello, si bien no ha habido investigaciones locales mostrando que la agresividad de esta enfermedad haya cambiado en Chile.

La distribución geográfica de residencia de los pacientes, similar a la de la población general en Chile, representa un estímulo para desarrollar programas de diálisis crónica en zonas distantes de la capital del país. En algunos casos se ha recurrido al manejo con médicos pediatras locales bajo supervisión a distancia.

La situación nutricional de estos niños causa preocupación. La uremia crónica afecta severamente el crecimiento longitudinal, especialmente si el trastorno renal se desartolla precozmen$\mathbf{t e}^{7}$. El bajo peso para la talla puede en parte explicarse por deficiente situación socioeconomica de la mayoría de los pacientes y probablemente por el tiempo de permanencia en DPC aún demasiado breve como para contrarrestar los efectos de la uremia severa no tratada sobre la nutrición.
La tếcnica quirúrgica en la colocación y cuidados postoperatorios del catéter fue similar en la mayoría de los centros consultados, en concordancia con iniciativas previas de estandarización. El uso sistemático de catéteres tipo Tenckhoff y su correcto manejo perioperatorio han contribuido probablemente en gran medida al buen exito obtenido con el procedimiento en los últimos años. Las diferencias en el tiempo transcurrido entre la instalación del catéter y el inicio de la DPC guardan relación con la urgencia de comenzar precozmente el procedimiento en algunos casos, por motivos clínicos o bioquímicos, aunque en condiciones menos adversas se aceptan las recomendaciones internacionales de hacerlo entre 10 y 14 días después ${ }^{9}$, 10 que se discutirá más adelante. La poca frecuencia del empleo en el país de DPC automatizada (considerada como la mejor opción en pediatría, ya que con su uso parecen menores las tasas de peritonitis ${ }^{10}$ ) se explica en parte por la falta de máquinas cicladoras y el mayor coste inicial y operacional que determinan.

La tasa de escolaridad en aquellos niños mayores de 6 años fue llamativamente buena, máxime tratándose de niños con riesgo de desarrollo neurológico alterado ${ }^{11}$, con importante ausentismo escolar por su enfermedad primaria en algunos casos, y con pobre situación socioeconómica en otros.

La tasa de peritonitis registrada en nuestro estudio, aunque comparable con otras de países iberoamericanos 12 , es todavía alta en comparación con la de Estados Unidos, donde es cercana a un episodio cada 13 pacientes/mes ${ }^{13}$ y en Europa, donde es todavía menor ${ }^{14}$. La alta tasa local podría radicar en la aún breve experiencia con el procedimiento, escasez de profesionales entrenados, una población compuesta principalmente de pacientes de baja situacion cultural y socioeconómica, y a la casi ausencia de DPC automatizada, todo lo cual aumenta el riesgo de contaminación del sistema ${ }^{10}$. El sitio de salida del catéter, a diferencia de lo descrito en una publicación reciente ${ }^{13}$, no se cortelaciono con mayor incidencia de peritonitis. El tipo de gérmenes causantes de las infecciones peritoneales y el porcentaje de ellas con cultivo de resultado negativo no difiere con lo publicado en la literatura ${ }^{15}$.

Si bien el tiempo transcurrido desde la incorporación al procedimiento de estos niños anali- 
zados es aún corto para obtener conclusiones sobre el buen éxito del método, es alentador observar que sólo un paciente debió ser retirado del programa y transferido a hemodiálisis, debido a inadecuada superficie peritoneal, producto de múltiples cirugías digestivas previas. La única muerte fue causada por una infección no relacionada a la diálisis y determina una proporción de letalidad semejante a la descrita en publicaciones internacionales ${ }^{13,14}$.

\section{Agradecimientos}

A todos los integrantes de la Rama de Nefrología de la Sociedad Chilena de Pediatria por responder en forma en. tusiasta y adecuada la encuesta enviada a sus centros.

\section{Referencias}

1. Diario Oficial de la Republica de Chile. 31 de diciembre de 1994: 36.

2. Diario Oficial de la Repüblica de Chile, 29 de diciembre de 1995: 30.

3. Saieh C. Bueza J. Cardero J, ef al: Dílisis peritoneal intermitente en pediatría. Rev Chil Pediatr 1986; 57 : $14\rfloor-145$

4. Gonzílez $F$, Gacs O. Fuentes de la $S, L$. Elgueta A: Diálisis peritoneal intermitente con cycler automático en el niño. Rev Pediatria Hospital Roberto del Río 1985; $28: 56-58$.

5. Valenauelo A, Lagomursino E. Cavagnaro F. Solar E: Insuficiencia renal crónica en la infancia. Encuesta nacional. Rev Chil Pediats 1986; 67; 116-120.
6. Valenzuela A. Lagomarsino E. Cavagnaro F, Solar E: Insuficiencia renal crónica pediátrica en Chile. Situación actual. Nefrología Latinoamericana 1996; 3; 226.

7. Schaefer $F$, Mehls $O$ : Endocrine. Metabolic and Growth Disorders, en Holliday M, Barrat M. Avner E. (eds.) Pediatric Neptrology, tercera edición, Williams $\&$ Wilkins, Baltimore, 1994; capítulo 62, 1241-1286.

8. Gokal $R_{1}$ Ash $S$, Helfrich B, el al: Periloneal catheters and exit-site practices: Toward Optimum Peritoneal Access. Peritoneal Dialysis Int 1993: 13: 29-39

9. Holloway M: CAPDICCPD Orders in Children, en Nissenson A y Fine R (eds.): Dialysis Therapy, segunda edicion, Hawley \& Belfus Inc. Philadelpbia. 1993.

10. De Fijter $C$, Verbrugh $H$, Oe $L$, el al: Peritoneal defense in continuous ambulatory versus continuous cyclic peritoneal diatysis. Kidney Int 1992; $42: 947$. 950.

11. Fine $R$, Salusky $Y$, Ettenger $R$ : The therapeutic approach to the infant, child, and adolescent with endstage renal disease. Pediatr Clin North Am 1987; 34 789-801.

12. Orso-Sibu $N$, Dominguez $L$, Scovino $R$, Moriyon $J$ Marcano $H$. Polanco $N: 10$ years experience with continuous ambulatory peritoneal dialysis (CAPD) in children in Valencio. Venezuela. Pediatr Nephrol 1995; 9:78.

13. Warady B. Sultivan EK, Alexander $S$ : Lessons from the peritoneal dialysis patient database: $A$ report of the North American Pediatric Renal Transplant Cooperative Study. Kidney Int 1996; 49 (Suppl 53): S68-7]

14. Edefonti A. Consalvo G. Pappalettera M: Infectious complications in pediatric patients treated with chronic peritoneal dialysis (CPD). Peritoneal Dialysis Int 1996; 16 (sup] 1): $\$ 543-547$.

15. Vas S: Peritonitis in peritoneal dialysis patients, en Nissenson A y Fine R (eds.): Dialysis Therapy, segunda edición Hawley \& Belfus Inc. Philadelphia, 1993.

\section{AVISO A LOS AUTORES}

Con el objeto de acelerar la edición de los trabajos se solicita encarecidamente a los autores que disponen de computador con procesadores de texto IBM compatibles, enviar -en lo posible junto con las dos copias impresas de su material-, otra en disco flexible (tamaño 3,5" o 5,25", de doble o alta densidad) del mismo texto. El disco será devuelto de inmediato por correo certificado o por mano al autor corresponsal. 